\title{
Removal of a Press-through Package in the Thoracic Esophagus Using two Flexible Endoscopes
}

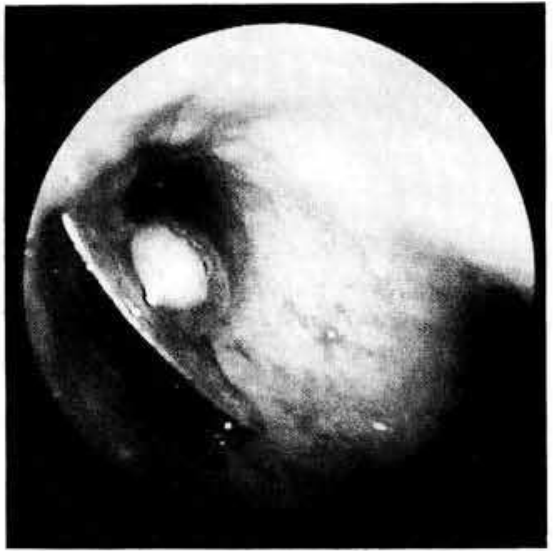

Figure 1:

Esophagoscopy

showing a press-

through package

(PTP) $(17 \times 18 \mathrm{~mm})$

in the thoracic

esophagus.

Flexible endoscopy is the primary examination for foreign bodies in the esophagus $(1,2)$. Although small foreign bodies can normally be extracted either with forceps or a snare, this may fail due to inadequate forceps or cause mucosal injury due to the sharp edges

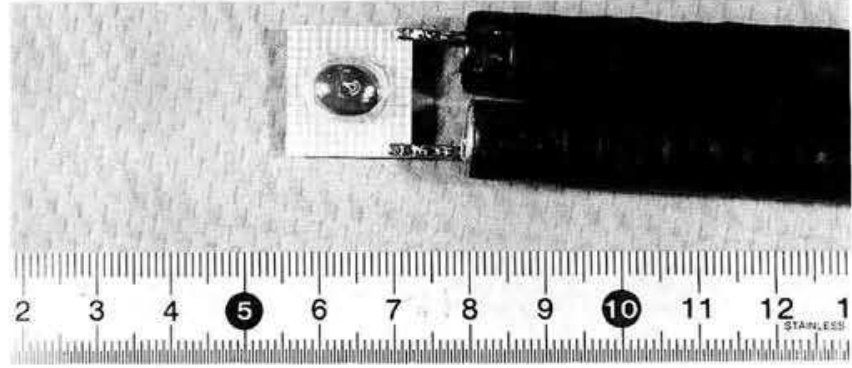

Figure 2: Two forceps grasp the bilateral oral edges of the PTP without causing injury to the esophageal mucosa during extraction.

for the foreign body. We herein present the removal of a pressthrough package (PTP), which has a flat shape with sharp edges, from the thoracic esophagus using two flexible endoscopes. The patient was a 79-year-old male who had swallowed a PTP by mistake. Panendoscopy showed a PTP $(17 \times 18 \mathrm{~mm})$ in the thoracic esophagus (Figure 1). Although we originally tried to remove the 
PTP with forceps, the PTP could not be extracted through the physiologic narrowing at the post-cricoid area and the sharp edges of PTP injured the esophageal mucosa. Thereafter, a second flexible endoscope was inserted. The oral edges of the PTP were bilaterally grasped by the two forceps (Figure 2) thereby removing the PTP easily. This method has three advantages: reinforcing of the strength of the forceps to hold the foreign body; a dilating effect on the physiologic narrowing at the post-cricoid area; and a lower risk of injuring the esophageal mucosa. This method could also be used for large foreign bodies which cannot be removed via an overtube (3). This safe, easy and less invasive method is therefore recommended for the removal of foreign bodies lodged in the thoracic esophagus as described in our case.

\section{Acknowledgement}

We thank B. Quinn for critical comments on the manuscript.
S. Tsutsui (1)*, T. Masuda (2), H. Matsuda ${ }^{(1)}$, N. Harada ${ }^{(2)}$, T. Sonoda ${ }^{(1)}$, K. Takada ${ }^{(2)}$

(1) Department of Surgery*

(2) Department of Internal Medicine

Saiseikai Fukuoka General Hospital

1-3-46 Tenjin, Chuo-ku

Fukuoka 810, Japan

\section{References}

1. Henderson $C T$, Engel J, Schlesinger $P$ : Foreign body ingestion: Review and suggested guidelines for management. Endoscopy 1987; 19: 68-71.

2. Webb WA: Management of foreign bodies of the upper gastrointestinal tract. Gastroenterology 1988; 94: 204-216.

3. Tedesco FJ: Endoscopic removal of foreign bodies using fiberoptic instruments. South. Med. J. 1977; 70: 991-994. 